\title{
HIPNOTERAPIA ERICKSONIANA
}

\author{
Ericksonian hypnotherapy
}

Jenny Quezada Zevallos*

\begin{abstract}
Resumen
La autora realiza una presentación de las principales características de la hipnoterapia ericksoniana, creada por Milton Hyland Erickson; inicia su artículo con una nota biográfica de M. Erickson, resaltando las tácticas interpersonales de abordaje del paciente, las cuales tienen como objetivo liberar las potencialidades para la autoayuda, tanto en estado hipnótico como en estado de vigilia.

La hipnoterapia de Erickson no sólo trabaja con técnicas específicas para tratar los síntomas, sino que trabaja y utiliza las actitudes, auto-imagen, flexibilidad conductual y emocional y la estructura familiar, factores que son importantes para un proceso de cambio más amplio.
\end{abstract}

Palabras clave: hipnoterapia, hipnoterapia Ericksoniana.

\begin{abstract}
The author does a presentation of the main characteristics of the Ericksonian Hypnotherapy, created by Milton Hyland Erickson. She begins her article with a biographical note about M. Erickson, pointing out the interpersonal tactics for approaching a patient, the ones that have as a goal to liberate the potentialities for self-helpness, both in hypnotic state as in wakefulness state.

Erickson Hypnotherapy does not only work with specific techniques for treating the symptoms, but it works and uses the attitudes, self-image, behavioral and emotional flexibility and family structure, important factors for a wider changing process.
\end{abstract}

Keywords: Hypnotherapy, Ericksonian hypnotherapy.

* Psicóloga. Docente Principal del Departamento de Psicología de la Universidad Femenina del Sagrado Corazón. jenquezada@unife.edu.pe 


\section{INTRODUCCIÓN}

En el año 1993 la autora del presente artículo realizó una formación en Hipnoterapia Ericksoniana, en el entonces Instituto de Hipnoterapia de Lima dirigido por el Dr. Lisle Sobrino y el Dr. Mario Pacheco, que abrió su mirada hacia las denominadas terapias no convencionales. Ahora bien, es frecuente la confusión de la identidad del psiquiatra Milton Hyland Erickson (1901-1980), connotado exponente de terapias no convencionales, con la del educador y psicoanalista Erik Erikson (1902-1994) por lo que se ha considerado necesario desarrollar en una primera parte la biografía del creador de la hipnoterapia ericksoniana.

El desarrollo de la hipnoterapia ericksoniana, posterior al fallecimiento de su creador, se ha visto enriquecida con los hallazgos provenientes de las investigaciones en neurofisiología, tales como: ritmos ultradianos; aprendizaje, memoria y comportamientos dependientes del estado (SDMLB); los estados atascados (statebound) y los transductores informacionales, los que han sido sistematizados por uno de sus más cercanos seguidores, Ernest Rossi; esta nueva concepción nos permite re-pensar y comprender desde otra perspectiva la relación mente-cuerpo y la enfermedad psicosomática.

Milton Hyland Erickson (Instituto para la Hipnosis Indirecta, 2000)

Nace en Aurum, Nevada (Estados Unidos de Norteamérica), el 5 de diciembre de 1901. A los 17 años acaba el bachillerato superior en Wishfield, desarrollando una parálisis infantil. La polio afectó su pierna y brazo del lado derecho. Entra en coma durante 15 días, del que sale comenzando su autorehabilitación. Desarrolló un tipo de concentración mental para cualquier movimiento mínimo, rehaciendo mentalmente cada movimiento repetidas veces. En cuanto adquiría más fuerza, aprovechaba cualquier oportunidad para ejercitar un nuevo grupo de músculos, para fortalecerlos cada vez más, aprendiendo a andar con muletas, a equilibrarse y a andar en su bicicleta. Al final del verano es capaz de moverse por sí solo e ingresa a la escuela de medicina.

En 1925 estando ya casado, termina la carrera de psiquiatría y empieza a trabajar en el Hospital del Estado de Rhode Island como Senior Psychiatric.
En 1934 es nombrado Director del departamento de investigación y formación psiquiátrica del Wayne Country General Hospital, Eloise, en Michigan, posteriormente es nombrado Profesor de psicología clínica en el Michigan State College.

De 1934 a 1948 trabaja como Director de Investigación Psiquiátrica en Eloise, posteriormente como Director Clínico del Hospital del Estado de Arizona, en Phoenix.

En la década de 1950 se forma el Grupo de Palo Alto en California, bajo la dirección teórica de Gregory Bateson y el liderazgo clínico de Don Jackson, el grupo de Palo Alto llegó a ser más tarde The Center for Brief Therapy of the Mental Research Institute of Palo Alto, más conocido como MRI. De sus estudios surge un nuevo enfoque de la comunicación humana destinado a producir un cambio epistemológico de enorme alcance. El contacto de Milton Erickson influyó poderosamente en el grupo.

En 1957 funda la Sociedad Americana de Hipnosis Clínica, junto con E. Aston, S. Hershman, W. Kroger, I. Secter y otros, siendo su primer presidente, para lo cual fundó y editó durante diez años su publicación oficial, The American Journal of Clinical Hypnosis.

Entre 1970 y 1980, él fue perdiendo gradualmente su fuerza muscular y un poco del control de los músculos de la lengua y las mejillas, perdió su capacidad de mantener la focalización ocular por largos períodos de tiempo y tuvo que abandonar su extensa actividad de lectura tanto profesional como recreativa. Erickson tenía una perspectiva positiva sobre su condición, él acostumbraba decir que la poliomielitis había sido su mejor profesor sobre el comportamiento humano (Zeig, 1980a).

Con la publicación del libro Terapia no convencional de Jay Haley (1973), Erickson se hizo más conocido como padre de los abordajes estratégicos breves para psicoterapia.

Sus alumnos Jeffrey Zeig y Ernest Rossi se dedicaron a realizar la recopilación de escritos, grabaciones y relatos de experiencias personales con Erickson en la Fundación Milton H. Erickson en Phoenix, Arizona, parte de cuyo material ha sido publicado. 
Milton Erickson fallece a los 79 años, en el año 1980, en Phoenix, Arizona.

\section{HIPNOTERAPIA}

Las técnicas de la hipnosis históricamente son iniciadas por Mesmer, ilustre médico alemán, en el siglo XVIII; posteriormente, en el siglo XIX, los trabajos con respecto a este tema fueron realizados por Charcot, Braid, Lièbeault y Bernheim; en el siglo XX Erickson, basándose en la moderna hipnosis médica, crea nuevos métodos de utilización e inducción hipnótica. Él fue el co-autor de cinco libros sobre el tema y publicó más de 130 artículos, la mayoría de ellos sobre hipnoterapia. La hipnosis ericksoniana difiere radicalmente de la hipnosis estandarizada, en su empleo de la utilización, dirección e intervenciones estratégicas.

La importancia de la denominada Psicología Ericksoniana radica tanto en las técnicas creadas como en la filosofía que está detrás de los métodos, así como las tácticas interpersonales de abordaje del paciente, las cuales tienen como objetivo liberar las potencialidades para la autoayuda, tanto en estado hipnótico como en estado de vigilia. Erickson es considerado un innovador en psicoterapia, al enfatizar en su método la utilización de recursos internos del propio paciente; muchos destacados profesionales fueron influenciados por sus ideas, por ejemplo: Margaret Mead, Gregory Bateson, Jay Haley, John Weakland, Ernest Rossi, Stephen Lankton y Joseph Barber.

Los discípulos más cercanos a Erickson han producido una creciente bibliografía sobre hipnosis clínica: Jeffrey Zeig (1980a, 1980b, 1987), Ernest Rossi (Erickson \& Rossi, 1979), Erickson \& Rossi, 1981; Erickson, 1983, 1985; Rossi, 1986a y 1986b; Rossi \& Ryan, 1986; Rossi \& Cheek, 1988), Stephen Lankton y C. Lankton (1983) y muchos otros. Zeig (1985) propone que:

La psicoterapia ericksoniana es un abordaje pragmático y estructural, basado en el diagnóstico y la modificación de patrones mal adaptados del paciente, promoviendo cambios, elucidando el pasado o promoviendo insights sobre el significado o la función de los síntomas. Para promover cambios basados en las necesidades más profundas del paciente o terapeuta adaptándose al contexto o a un cuadro de referencia del mismo, utilizando un patrón de comunicación terapéutica individualizada y de múltiples niveles, para identificar, despertar, desenvolver, recombinar y utilizar los recursos del propio paciente. (p. 24)

Siguiendo al mismo autor, en el concepto ericksoniano no existe mucha diferencia entre hipnosis, enseñanza o psicoterapia, porque en todas estas áreas se debe creer en el aprendizaje inconsciente, el cual permitiría el desenvolvimiento de los recursos, los cuales combinados de una manera nueva y más eficiente, posibilitarán procesos de salud.

Erickson y Rossi (1979), referidos por Pacheco (1990a), definen al trance terapéutico como un período en el cual los pacientes son capaces de romper sus estructuras y sistemas de creencias, mientras pueden experimentar otros patrones de funcionamiento dentro de sí mismos. El proceso de trance es descrito en cinco fases: 1) Fijación de la atención, 2) Debilitación de los marcos habituales y marcos de creencias, 3) Búsqueda inconsciente, 4) Procesos inconscientes y 5) Respuesta hipnótica.

Haley (1970), referida por Bowie (1990a), explica que Erickson siempre veía a su paciente en el contexto de su vida relacional, en su familia o grupo social.

El quería saber de sus pacientes: ¿cómo están tus relaciones, con quienes vives y trabajar?, ¿cuál es tu meta para mañana y pasado?, ¿Dónde estás ahora con respecto a esa meta?, ¿qué te impide llegar?, ¿qué recursos tienes para ayudarte a llegar? Luego Erickson se preguntaba: ¿qué puedo hacer yo como terapeuta para facilitar la captación de esos recursos que el paciente ya tiene dentro de sí, $\mathrm{y}$ asociarlos a sus vivencias reales de tal manera que éstos puedan ayudarle a alcanzar la meta anhelada?, ¿qué obstáculos necesita superar?. Su metodología era la de fortalecer lo bueno que estaba haciendo su paciente, en lugar de analizar sus deficiencias o preguntar el por qué de esas limitaciones. Si el paciente carecía de recursos, Erickson buscaba con increíble tenacidad cómo desarrollarlos, como en el caso del "February man” (Erickson y Rossi, 1989). (pp. 4-5).

La principal herramienta del método ericksoniano es el nivel de comunicación psicológica indirecta 
(Lankton, Lankton \& Brown, 1981; Lankton \& Lankton, 1983, referidos por Zeig, 1985). Erickson notó que la comunicación sucede en múltiples niveles, incluyendo el contenido verbal, el comportamiento no verbal y las implicaciones de cada una de ellas: el método indirecto es un proceso por el cual las respuestas ocurren sin que el sujeto tenga completa consciencia de esto. Algunas de las formas que Erickson usaba para ser más indirecto era contando historias, anécdotas, analogías y proverbios para ejercer influencias en múltiples niveles. La consciencia de los múltiples significados de las palabras era la piedra angular de su técnica indirecta.

Erickson combinaba sus conocimientos con la utilización de los valores propios del paciente (cosmovisión) para dirigir asociaciones internas y promover cambios en el contexto, hasta que hubiese un suficiente ayuda de asociaciones que permitiese al paciente efectuar por sí mismo y voluntariamente los cambios necesarios.

Zeig (1985) plantea que para que para que un terapeuta realice una comunicación transformadora a nivel psicológico, esta requiere ser obtusa, indirecta, metafórica, ilógica y puede consistir a veces en tareas estratégicas aparentemente irrelevantes.

Erickson, en vez de discutir de técnicas específicas, prefería abordar la idea de "utilización", la cual significa básicamente que las mejores técnicas son aquellas que se derivan de los propios pacientes y no del terapeuta; en una especie de "traje a la medida del paciente", para lo cual utilizaba un abordaje flexible que podía incluir interpretación, sugestión indirecta o hipnosis.

Zeig (1985) plantea los siguientes aspectos importantes para que el abordaje utilizacional funcione:

1. Identificar los recursos latentes del paciente.

2. Identificar los valores del paciente (tanto lo que más le gusta como lo que no le gusta).

3. Desarrollar tales recursos utilizando los valores del propio paciente.

4. Vincular el recurso desarrollado al problema tanto directa como indirectamente.
5. El cuarto paso será mejor concretizado cuando se haga en pequeños pasos, consiguiendo la confianza del paciente, estableciendo rapport, creando motivación y controlando la responsividad a lo largo del proceso.

6. Cualquier comportamiento, cualquier manifestación, cualquier resistencia deben ser aceptados y pueden ser utilizados como herramienta terapéutica.

7. El drama puede ser utilizado para mejorar la responsividad a las directivas.

8. Sembrar las ideas antes de presentarlas genera comportamiento responsivo.

9. El ritmo es un factor crucial, mas el proceso terapéutico implica también pacing, distanciamiento.

10. El terapeuta (y el paciente) debe tener una actitud de expectativa.

11. Acompañamiento de los resultados.

La hipnoterapia de Erickson no sólo trabaja con técnicas específicas para tratar los síntomas, sino que trabaja y utiliza las actitudes, auto-imagen, flexibilidad conductual y emocional y la estructura familiar, factores que son importantes para un proceso de cambio más amplio.

\section{DIFERENCIAS ENTRE LA HIPNOSIS ERICKSONIANA Y LA HIPNOSIS TRADICIONAL}

Basándonos en el artículo de Miserda (1990) realizaremos un resumen de la propuesta de las diferencias entre la hipnosis Ericksoniana y la hipnosis tradicional, partiendo de que no son dos tipos diferentes de hipnosis, ya que sólo hay una hipnosis, es decir el trance hipnótico, en el cual los mismos mecanismos psicofisiológicos están en la base de ambas.

A continuación algunas de sus diferencias:

1. El enfoque tradicional de la hipnosis (H.T.) considera al trance como un fenómeno un tanto artificial, que es provocado por el "poder" del hipnotista en algunos sujetos susceptibles.

En el enfoque ericksoniano (H.E.), en cambio, se considera al trance como un fenómeno natural que experimentamos todas las 
personas cotidianamente, como expresión del funcionamiento neurofisiológico, y que puede ser evocado por el hipnotista adiestrado.

2. La H.T considera en algún modo el trance como un estado de vulnerabilidad psicológica para un mejor cumplimiento de las sugestiones directas del hipnotista.

La H.E. considera el trance como un estado que rodeando las limitaciones conscientes aprendidas del sujeto, le permite experimentar otros patrones de funcionamiento previamente aprendidos, pero no disponibles conscientemente.

En dicho estado se alcanza una mejor predisposición para el aprendizaje y una utilización más eficaz de las potencialidades y creatividad del sujeto.

3. En la H.T. la inducción de trance generalmente se consigue a través de procedimientos más ritualísticos y estándares (uso de péndulo, metrónomo, objetos delante de los ojos, etc.).

En la H.E. la evocación del trance se consigue con procedimientos más naturales y distintos para cada sujeto (una conversación interesante, estrechando la mano, fijación de la mirada, etc.).

4. LaH.T.postulaquelafacilidadparaexperimentar trance es debida a la "hipersugestibilidad" del sujeto, encontrándose sujetos refractarios o resistentes a la hipnosis.

La H.E. no considera a la "hipersugestibilidad" como un requisito para entrar en trance. La observación cuidadosa del sujeto y la utilización creativa de todo su comportamiento -incluida la resistencia- permitirá ayudarlo a entrar en trance.

5. En la H.T. los métodos ritualísticos de inducción obligan al sujeto a adaptarse a las sugerencias del hipnotista, las cuales generalmente son autoritarias y rígidas.

En la H.E. por el contrario, es el hipnotista quien va adaptando sus sugerencias al comportamiento presente del sujeto; lo cual crea un clima de aceptación y una relación interpersonal con retroalimentación mutua (rapport). Se utilizan métodos libres de fracaso, tanto para el sujeto como el hipnotista.
6. En las H.T. las sugestiones son directas, autoritarias y repetitivas.

En la H.E. las sugestiones son indirectas, flexibles y se van modificando según las respuestas del sujeto.

7. El cambio que provoca la H.T., es un proceso impuesto por el hipnotista, una especie de "hetero-injerto psicológico", variando su efectividad según el tipo de sujeto.

8. La H.E al utilizar sugestiones indirectas favorece una síntesis y resíntesis interna en el sujeto, promoviendo un cambio con el máximo de libertad, utilizando la creatividad y recursos del sujeto; por lo cual éste cambia a "su manera" (como un "auto-injerto psicológico").

9. La H.T. puede afectar la autoestima del paciente, el cual puede sentirse manipulado y subordinado.

La H.E. puede afectar la autoestimación del sujeto, al participar éste más activa y libremente en el proceso de trance.

10. La H.T. requiere de la habilidad para conocer y aplicar ritualísticamente las estrategias de inducción de trance.

La H.E. requiere y promueve la creatividad del hipnotista, ya que éste necesitará desarrollar su capacidad de observación y flexibilidad para adaptarse a cada paciente como un individuo único.

\section{NUEVOS FUNDAMENTOS CIENTÍFICOS DE LA HIPNOSIS}

Ernest Rossi, colaborador de Milton Erickson y editor de los escritos y trabajos clínicos de Erickson, ha realizado una síntesis de los nuevos adelantos científicos, entregando un modelo de hipnosis que da validez a su poder y utilidad como instrumento clínico.

En sus libros de The psychobiology of mindbody healing: New concepts of therapeutic hypnosis (1986a) y Mind-Body therapy: Ideodinamic healing in hipnosis (Rossi y Cheek, 1988) plantea que la información entre la mente y el cuerpo se traspasa a través del proceso de transducción y que todos los problemas humanos pueden concebirse como bloques que obstaculizan el flujo de información entre y dentro de la mente, cuerpo y sociedad. Cuando hay una falta 
de información, nuestras capacidades de ajuste y adaptación fallan y nos sentimos presionados por el "stress". Es aquí donde Rossi, integra el concepto de GAS de Hans Selye, el cual propone que los síntomas psicosomáticos se adquieren por medio de un proceso de aprendizaje experiencial, el "stress" acumulado y agravado se asocia con los trastornos psicofisiológicos o "psico-somáticos". A este concepto se le ha dado el nombre de "State-Depend Memory, Learning and Behavior" (SDMLB), (traducido sería: Memoria, Aprendizaje y Comportamiento Dependientes del Estado). La hipnoterapia consiste en un proceso que da acceso a la información "atascada en su estado" y liberarla para su uso en la resolución de los problemas.

Pacheco (1990a, 1990b) sostiene que los hallazgos provenientes de las investigaciones en neurofisiología, tales como: ritmos ultradianos; aprendizaje, memoria y comportamientos dependientes del estado (SDMLB); los estados atascados (statebound) y los transductores informacionales (Rossi, 1982, 1986; Rossi y Cheek, 1988), nos permiten re-pensar y comprender desde otra perspectiva la relación mente-cuerpo y la enfermedad psicosomática.

\section{REFERENCIAS}

Bowie, Dorothy (1990a). La hipnosis clínica ericksoniana. En Mario Pacheco, Introducción a la hipnoterapia ericksoniana (pp. 1-12), Santiago de Chile: Instituto Chileno de Hipnosis Aplicada.

Bowie, Dorothy (1990b). La hipnosis: nuevos fundamentos científicos. En Mario Pacheco, Introducción a la hipnoterapia Ericksoniana (pp. 64- 67), Santiago de Chile: Instituto Chileno de Hipnosis Aplicada.

Instituto para la Hipnosis Indirecta. (2000). Biografía Milton H. Erickson. Recuperado de http://www. paginasprodigy.com/hipnosis-capaf/Biograf $\% \mathrm{C}$ $3 \%$ ADa\%20Milton\%20H_\%20Erickson\%20$\% 20 \mathrm{IHI} . \mathrm{htm}$

Erickson, M. H. (1983). Healing in hypnosis. En Ernest L. Rossi, Margaret O. Ryan \& Florence A. Sharp (Eds.), The seminars, workshops, and lectures of Milton H. Erickson (Vol. I). New York: Irvington Publishers.

Erickson, M. H. (1985). Life reframing in hypnosis. En Ernest L. Rossi; Margaret O. Ryan and Florence A. Sharp (Eds.), The seminars, workshops, and lectures of Milton H. Erickson (Vol. II). New York: Irvington Publishers.

Erickson, M. H. \& Rossi, E. L. (1979). Hypnotherapy: an exploratory casebook. New York: Irvington Publishers.

Erickson, M. H. \& Rossi, E. L. (1981). Experiencing hypnosis: Therapeutic approaches of altered states. New York: Irvington Publishers.

Erickson,H.M. \& Rossi,E.L.(1989). The februaryman: evolving consciousness and identit in hypnotherapy. New York: Bruner / Mazel Publishers.

Haley, J. (1973). Uncommon therapy: The psychiatric techniques of Milton Erickson, MD. New York: W.W. Norton.

Lankton, S. \& Llankton, C. (1983) The answer within: a clinicalframework of Ericksonian hypnotherapy. New York: Brunner / Mazel Publishers.

Miserda, R. (1990). Diferencias entre la hipnosis ericksoniana y la hipnosis tradicional. En Mario Pacheco, Introducción a la hipnoterapia ericksoniana (pp. 8 - 12), Santiago de Chile: Instituto Chileno de Hipnosis Aplicada.

Pacheco, Mario. (1990a). Fases de la hipnoterapia ericksoniana: microdinámicas de la sugestión hipnótica. En Mario Pacheco, Introducción a la hipnoterapia ericksoniana (pp. 36-41), Santiago de Chile: Instituto Chileno de Hipnosis Aplicada.

Pacheco, Mario. (1990b). Introducción a la hipnoterapia ericksoniana. Santiago de Chile: Instituto Chileno de Hipnosis Aplicada.

Rossi, E. L. (1982). Hypnosis and ultradian cycles: a new state(s) theory of hypnosis? American Journal of clinical hypnosis, 25, 21-32.

Rossi, E. L. (1986a). The psychobiology of Mind-Body healing: New concepts of therapeutic hypnosis. New York: W.W. Norton.

Rossi, E. L. (1986b). Altered states of consciousness in everyday: the ultradian rhythms. En B. Woman (Ed.), Handbook of altered states of consciousness. New York: W.W. Norton. 
Rossi, E. L. \& Ryan, M. O. (1986). Mind-body communications in hypnosis. The seminars, workshops, and lectures of Milton H. Erickson. New York: Irvington Publishers.

Rossi, E. L. \& Cheek, D. B. (1988). Mind-body therapy: ideodinamic healing in hypnosis. New York: W.W. Norton.

Zeig, J.K. (1980a). A teaching seminar with Milton $H$. Erickson. New York: Brunner/ Mazel.

Zeig, J.K. (1980b). Symptom prescription and ericksonian principles of hipnoisis and psychotherapy. American Journal of Clinical Hypnosis, 23, $16-22$

Zeig, J. K. (1985). Vivenciando Erickson. Uma apresentação da pessoa humana e do trabaho de Milton H. Erickson, M.D. Campinas: Psy

Zeig, J. K. (1987). Therapeutic patterns of Ericksonian influence communication. In J. K. Zeig, The evolution of psychotherapy (pp. 392-406), New York: Brunner/ Mazel. 